\title{
Folded Waveguide Traveling-Wave Tube Sources for Terahertz Radiation
}

\author{
Sudeep Bhattacharjee, Member, IEEE, John H. Booske, Senior Member, IEEE, Carol L. Kory, Member, IEEE, \\ Dan W. van der Weide, Member, IEEE, Steve Limbach, S. Gallagher, John D. Welter, Mike R. Lopez, Member, IEEE, \\ Ronald M. Gilgenbach, Senior Member, IEEE, R. Lawrence Ives, Senior Member, IEEE, \\ Michael E. Read, Member, IEEE, Ralu Divan, and D. C. Mancini
}

\begin{abstract}
Microfabricated folded waveguide traveling-wave tubes (TWTs) are potential compact sources of wide-band, high-power terahertz radiation. We present feasibility studies of an oscillator concept using an amplifier with delayed feedback. Simulations of a 560-GHz oscillator and experimental evaluation of the concept at $50 \mathrm{GHz}$ are presented. Additionally, results from various fabrication methods that are under investigation, such as X-ray lithography, electroforming, and molding (LIGA), UV LIGA, and deep reactive ion etching are presented. Observations and measurements are reported on the generation of stable single-frequency oscillation states. On varying the feedback level, the oscillation changes from a stable single-frequency state at the threshold to multifrequency spectra in the overdriven state. Simulation and experimental results on amplifier characterization and dynamics of the regenerative TWT oscillator include spectral evolution and phase stability of the generated frequencies. The results of the experiment are in good agreement with the simulations.
\end{abstract}

Index Terms-Delayed feedback oscillator, folded waveguide (FWG) traveling-wave tube (TWT), frequency spectrum, high power, microfabrication, phase, terahertz radiation, wide-band.

\section{INTRODUCTION}

$\mathbf{T}$ HE TERAHERTZ $(\mathrm{THz})$ region of the electromagnetic spectrum ( $\sim 300-3000 \mathrm{GHz}$ in frequency or $\sim 0.1-1.0-\mathrm{mm}$ free space wavelength) has enormous potential for high data rate communications, advanced electronic materials spectroscopy, space research, medicine, biology, surveillance, and remote

Manuscript received September 20, 2003; revised February 10, 2004. This work was supported in part by the U.S. Air Force Office of Scientific Research, the Office of Naval Research (YIP), the Innovative Microwave Vacuum Electronics Multidisciplinary University Research Initiative (MURI) program, managed by the U. S. Air Force Office of Scientific Research and Air Force Small Business Technology Transfer, under Contract F4920-99-C-0064-DEF. The work at Argonne National Laboratory was supported by the U.S. Department of Energy under Contract W-31-109-ENG-38.

S. Bhattacharjee, J. H. Booske, D. W. van der Weide, S. Limbach, S. Gallagher, and J. D. Welter are with the Department of Electrical and Computer Engineering, University of Wisconsin, Madison, WI 53706 USA (e-mail: sudeepb@ece.wisc.edu).

C. L. Kory is with the Analex Corporation, NASA Glenn Research Center, Cleveland, OH 44135 USA.

M. R. Lopez and R. M. Gilgenbach are with the Department of Nuclear Engineering and Radiological Sciences, University of Michigan, Ann Arbor, MI 48105 USA.

R. L. Ives and M. E. Read are with Calabazas Creek Research, Inc., Saratoga, CA 95070 USA.

R. Divan and D. C. Mancini are with Advanced Photon Source, Argonne National Laboratory, Argonne, IL 60439 USA.

Digital Object Identifier 10.1109/TPS.2004.828886 sensing [1]. However, this region of the spectrum still remains one of the most unexplored. Using carrier frequencies above $300 \mathrm{GHz}$, oscillator and amplifier sources with $\sim 10 \%$ fractional bandwidths would enable very high data rate $(>10 \mathrm{~Gb} / \mathrm{s})$ wireless communications with high-security protection. The shorter wavelengths at these frequencies allow the use of smaller and lighter components, which is important in military and space-borne applications, where size and weight are a prime concern. In addition, the atmospheric attenuation of millimeter and submillimeter waves is relatively low compared with infrared and optical wavelengths [2]. Adequate power sources in the THz regime would also enable imaging of biological tissue, where specific absorption rates (SARs) are large and, hence, require more power.

However, the critical barrier to full exploitation of the $\mathrm{THz}$ band is the lack of compact, powerful $(1-1000 \mathrm{~mW})$, coherent radiation sources that are efficient $(\geq 1 \%)$, frequency agile (instantaneous bandwidth $\geq 1 \%$ ), reliable, and comparatively inexpensive [3]. Gas lasers are candidate sources of coherent power at the upper end of this frequency range. However, they have small bandwidths and probably have limited applicability particularly in airborne or space-borne systems because of size and weight considerations [4]. As a spectroscopic source, they are relatively expensive. Quantum cascade lasers [5] can be designed to emit radiation over a wide range of wavelengths, and they outperform diode lasers in terms of power and current handling capability. Solid-state devices such as Gunn or IMPATT diodes can produce $1-10 \mathrm{~mW}$ of power at $300 \mathrm{GHz}$ [6]. They have very low output power at frequencies higher then $300 \mathrm{GHz}$ due to mobility limits on electrons. To operate at higher frequencies, frequency multipliers may be used [7]. However, the multiplication efficiency of the device decreases with increasing harmonic number, thereby producing low output power at low efficiency.

Quasi-optical arrays using solid-state sources are another option. They are currently being researched at microwave and millimeter-wave frequencies [8]. Typical fast wave vacuum electron devices in the $\mathrm{THz}$ regime such as gyrotrons [9] and free electron lasers (FELs) [10] tend to be large and expensive and require high voltage, unsuitable for most of the applications cited above. THz regime klystrons are being examined at the Jet Propulsion Laboratory, Pasadena, CA [11], and the University of Leeds, Leeds, U.K. [12], and transit time oscillators at the University of Michigan, Ann Arbor [13]. Currently marketed grating-based backward-wave oscillators (BWOs) are good 
for spectroscopy applications, but are not readily adapted for applications involving rapid signal modulation. In general, BWOs have relatively narrow instantaneous bandwidths, are heavy, require bulky magnets, have low efficiencies, and do not provide an amplifier configuration option [14], [15]. THz-regime cross-field amplifiers would have an unrealistically high magnetic field requirement. Traveling-wave tubes (TWTs) have demonstrated capabilities consistent with $\sim 100 \mathrm{~W}$ at $100 \mathrm{GHz}$ [16]. Also they would appear to provide substantial bandwidths. However, other than one or two rare instances [17], they have received very little study as candidate sources of radiation above $100 \mathrm{GHz}$.

To address the need and issues for THz-regime sources, we are researching the prospects for microfabricated folded waveguide (FWG) TWTs [18]-[20]. Microfabricated vacuum electron devices ( $\mu$ VEDs) are a blending of available technologies in conventional vacuum and solid-state electronics. It is expected that some of the limitations on circuit size and precision three-dimensional (3-D) fabrication which arise in vacuum electronics, and electron mobility and heat transfer encountered in solid-state electronics, will be overcome.

This paper describes studies of FWG TWTs for THz amplifiers and oscillators. A conceptual design of a $560-\mathrm{GHz}$ amplifier is first examined in Section II in order to identify feasible amplifier performance specifications and representative circuit dimensions and electron beam parameters. Subsequently, in Sections II-V, the article examines in greater detail the characteristics of a $\mathrm{THz}$ regime regenerative oscillator by combining a forward-wave TWT amplifier with delayed feedback. Issues of interest to the investigation of this oscillator configuration include: 1) the prospects for stable, single-frequency operation; 2) identification of physical parameters with promising potential for signal modulation and control; 3) instantaneous bandwidth available for modulated signal transmission; and 4) an assessment of technologies available for circuit fabrication. Therefore, the second part of Section II discusses numerical simulations of a $560-\mathrm{GHz}$ regenerative FWG TWT oscillator. Section III describes the design of a scaled experiment at $50 \mathrm{GHz}$ to acquire information on the first three issues cited above related to this particular oscillator configuration. Between the $50-\mathrm{GHz}$ and $\mathrm{THz}$ oscillator, the periodicity, transverse dimensions, and, to a lesser extent, the circuit length scales as the free space wavelength. Section IV presents and discusses the experimental results. Results of research into various circuit fabricating technologies are discussed in Section V. Finally, the paper is summarized in Section VI.

\section{AmPLifIER AND OSCILlator Design AT $560 \mathrm{GHz}$}

Designing FWG TWTs to meet gain, bandwidth, power, and operating frequency band specifications is nontrivial due to the dispersive properties of the circuit, the effect of beam space charge on the gain and operating bandwidth, and the effect of ohmic wall losses, which are significant at THz-regime frequencies. To realize an efficient and reliable design approach, we have coordinated the use of several recently developed computational tools: MAFIA, HFSS, TWA3, and CHRISTINE.

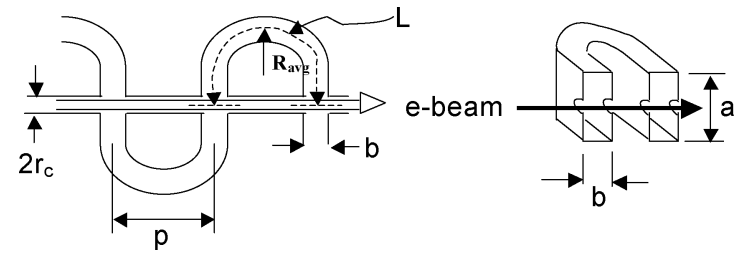

Fig. 1. FWG circuit sketch. Indicated are the transverse dimensions, $a$ and $b$ of the rectangular guide, the beam hole radius $r_{c}$, the average radius $R_{\text {avg }}$, and period $p$ of the serpentine structure.

TABLE I

Dimensions of 560-GHz FWG SLOW-WAVE CIRCUIT

\begin{tabular}{cc}
\hline \hline Parameter & Dimension $(\mu \mathrm{m})$ \\
\hline $\mathrm{b}$ & 43 \\
$\mathrm{a}$ & 300 \\
$\mathrm{R}_{\text {avg }}$ & 33 \\
$\mathrm{~L}$ & 32 \\
$\mathrm{r}_{\mathrm{c}}$ & 10 \\
$\mathrm{p}$ & 66 \\
\hline \hline
\end{tabular}

Slow-wave circuit propagation properties are obtained using the 3-D simulation codes MAFIA [21] and HFSS [22] and used as input into the TWT interaction codes TWA3 [23] and CHRISTINE [24] to determine gain and power transfer characteristics. In addition, the time-domain, particle-in-cell simulation feature of MAFIA has allowed us to conduct interaction simulations as well as qualitative examinations of transient startup and frequency spectra in THz-regime oscillators that utilize a FWG TWT as the active gain part of the device.

Applying the suite of computational models, we have developed realistically achievable designs for a $560-\mathrm{GHz}$ amplifier and a delayed feedback oscillator. A schematic of the FWG TWT circuit is shown in Fig. 1. The dimensions and representative geometry are shown in Table I. In the design, several options for power feedback to achieve oscillation with a forward-wave TWT were considered. Calculations revealed that a Fabry-Pérot approach of reflecting the forward power backward along the FWG circuit is impractical due to high ohmic losses suffered by the reflected wave at $\mathrm{THz}$ frequencies. Moreover, any significant advantage of such a means to produce an oscillator over the BWO configuration was not apparent. However, recirculating a fraction of the forward wave back to the input of the circuit is a viable option. To minimize unintended loss in the recirculated power leg of actual devices one may up-taper the small lateral dimension of the rectangular waveguide. The dispersion diagram is shown in Fig. 2 and the on-axis interaction impedance and attenuation (wall conductivity was assumed to be about $4 \times 10^{7} \mathrm{~S} / \mathrm{m}$ ) are shown in Fig. 3 .

Fig. 4 shows the results of a calculation of the small-signal gain for a short, 6.6-mm-long section of the circuit, assuming a 10.9-kV 0.5-mA electron beam. The gain was calculated with several different computational models and compared. The MAFIA simulations were done for a lossless circuit only, but the agreement with the parametric code TWA3 is excellent. Furthermore, both TWA3 and CHRISTINE agree in predicting approximately 10-dB gain between 520 and $580 \mathrm{GHz}$ (a 10\% 


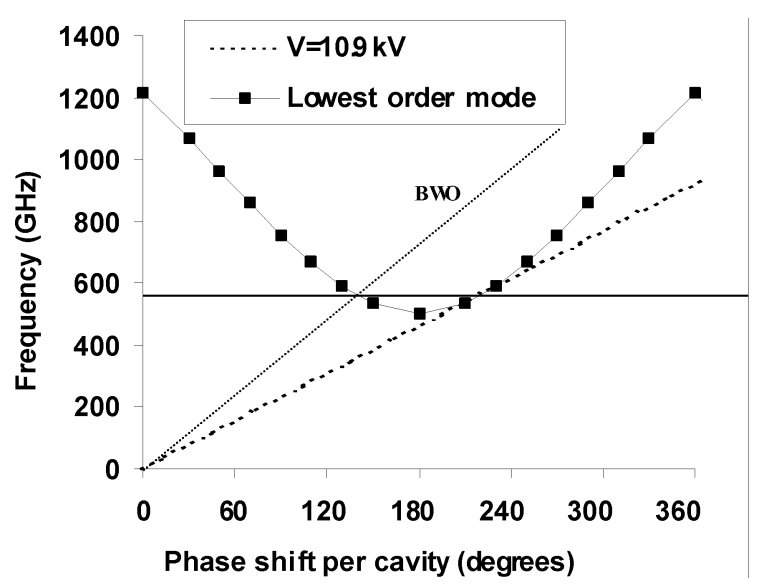

Fig. 2. Simulated dispersion for a forward-wave TWT amplification using a FWG circuit with a $10.9-\mathrm{kV}$ beamline (short dash). For reference, a backward-wave interaction is also indicated (long dash is higher voltage beam line).

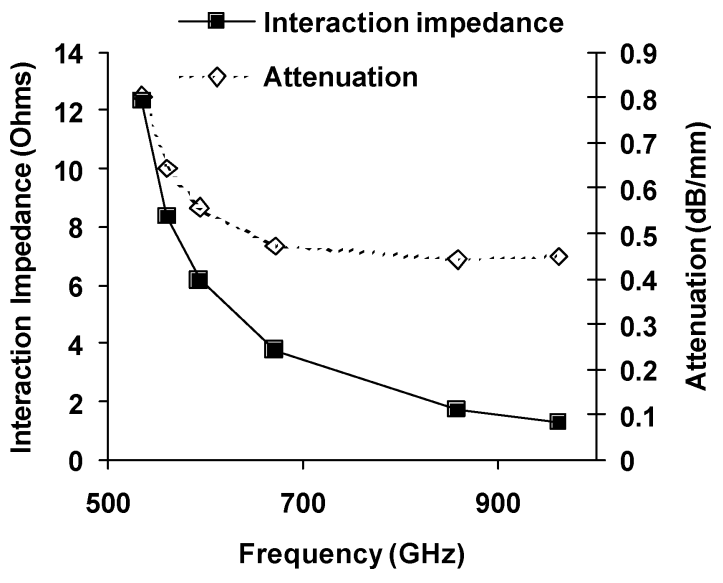

Fig. 3. MAFIA simulated on-axis interaction impedance and attenuation for FWG circuit.

fractional instantaneous bandwidth) when wall losses are included.

To investigate achievable levels of output power, large-signal simulations were also undertaken. For this example, a 2.5-cm-long circuit was predicted using CHRISTINE to produce $\sim 23 \mathrm{~dB}$ of gain at $560 \mathrm{GHz}$. Saturated output power was predicted to be $\sim 73 \mathrm{~mW}$, as shown in Fig. 5. Analytic estimates of the losses experienced in the recirculation leg combined with the predicted forward gain lead to an approximate prediction of steady-state radiated power of $\sim 56 \mathrm{~mW}$ at $560 \mathrm{GHz}$. This represents an intrinsic efficiency of $1 \%$, assuming stable oscillator operation can occur near saturated single-pass amplification condition. It should be noted that increased device efficiency and decreased waste heat dissipation at the beam collector could be expected from the use of a depressed voltage collector.

\section{A. Simulation of Feedback Oscillations}

The simulation code MAFIA was used for the investigation of frequency evolution with variable feedback. The simulations were carried out for oscillator circuit operating parameters as listed in Table II. Extensive computation times necessitated several compromises in the simulations: 1) a small length of

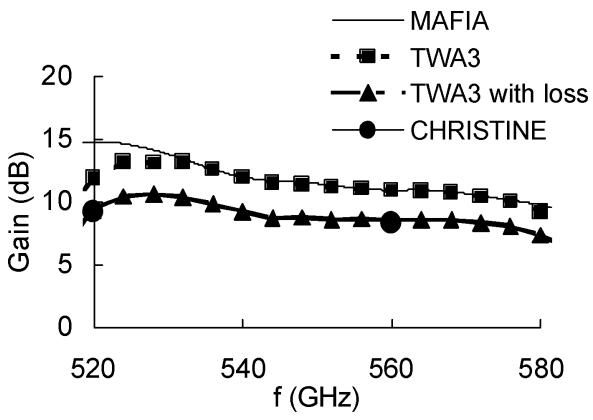

Fig. 4. Comparison of gain predictions for a 6.6-mm-long FWG TWT designed to operate at $\sim 560 \mathrm{GHz}$ using MAFIA (lossless), TWA3 (lossless and lossy), and CHRISTINE (lossy). The simulation assumes a 10.9-kV 0.5 -mA electron beam.

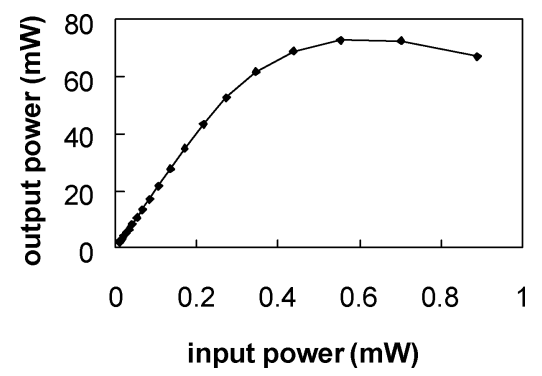

Fig. 5. Drive curve predicted by CHRISTINE for a $2.5-\mathrm{cm} 560-\mathrm{GHz}$ FWG TWT amplifier and $10.9-\mathrm{kV} 0.5-\mathrm{mA}$ electron beam. Saturated power is about $73 \mathrm{~mW}$.

TABLE II

OPERATING PARAMETERS OF THE $560 \mathrm{GHz}$ FOLDED WAVEGUIDE OSCILLATOR FOR MAFIA SIMULATIONS

\begin{tabular}{cc}
\hline \hline Quantity & Value \\
\hline Beam current $(\mathrm{mA})$ & 0.5 \\
Beam voltage $(\mathrm{kV})$ & 10.9 \\
Axial magnetic flux density $(\mathrm{T})$ & 0.67 \\
Beam radius /beam tunnel radius $\left(\mathrm{r}_{\mathrm{b}} / \mathrm{r}_{\mathrm{c}}\right)$ & 0.5 \\
\hline
\end{tabular}

the circuit was considered which provided a forward gain of approximately $9.75 \mathrm{~dB}$ and 2) the length of the simulation time was limited by allowing the oscillator to run for a maximum of approximately nine full round-trip transits of the electromagnetic energy. From the dispersion curve for the slow-wave circuit, we know that an RF wave propagating at $560 \mathrm{GHz}$ will have a phase velocity normalized to the speed of light, or $v_{p / c}$, approximately equal to 0.2 . A point of constant phase on the same RF wave will travel in the recirculation leg at approximately $1.8 c$ as a $\mathrm{TE}_{10}$ wave. Thus, the total time for a constant phase point of this wave to propagate the entire structure $t_{p}$ can easily be estimated as listed in Table III (cf. Fig. 6).

Next, attenuation in the recirculation leg was modeled. The small-signal gain of the FWG circuit length is about $9.75 \mathrm{~dB}$; thus, we included $8 \mathrm{~dB}$ of loss so as not to quench oscillation completely. The loss was simulated by filling a portion of the rectangular waveguide in the recirculation leg with dielectric having a finite conductivity of $0.5 \mathrm{~S} / \mathrm{m}$. Initial simulations of the recirculation leg without the FWG circuit were carried out to determine the value of conductivity providing the desired loss, 
TABLE III

560-GHz SimUlated Folded WAVEGUIDE OsCILLATOR PARAMETERS

\begin{tabular}{lc}
\hline \hline Quantity & Value \\
\hline $\mathrm{L}_{\text {tot }}(\mathrm{cm})$ & 1.01 \\
$\mathrm{~L}_{\mathrm{wg}}(\mathrm{cm})$ & 0.055 \\
Number of folded waveguide cavities & 154 \\
Approximate time to complete one complete path, $\mathrm{t}_{\mathrm{p}}(\mathrm{ns})$ & 0.19 \\
Total simulation time $\mathrm{t}_{\text {tot }}(\mathrm{ns})$ & 1.71 \\
Average number of path periods, $\mathrm{n}_{\mathrm{tp}}$ & 9 \\
\hline
\end{tabular}

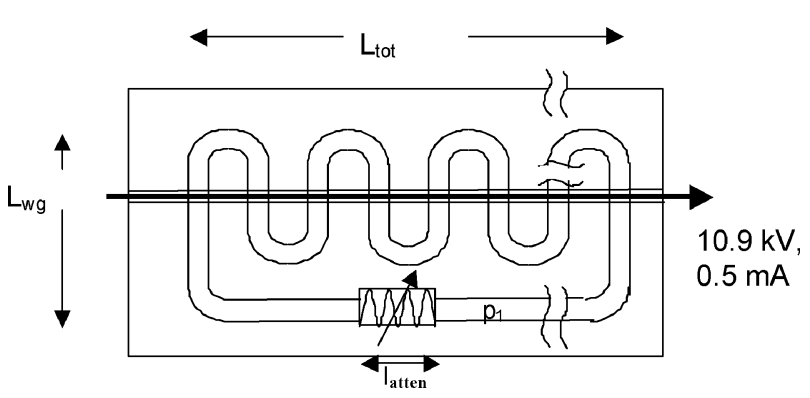

Fig. 6. Schematic illustration of simulated 560-GHz FWG TWT oscillator.

without introducing significant reflections due to mismatch between the vacuum and dielectric region in the rectangular waveguide. By performing $S$-parameter simulations, we found that filling the waveguide with this material provided $1837.85 \mathrm{~dB} / \mathrm{m}$ of loss at the center frequency of $560 \mathrm{GHz}$ without causing significant reflections. Thus, we defined the lossy section with a length of $4.354 \mathrm{~mm}$ to obtain $8 \mathrm{~dB}$ of attenuation. The length of dielectric $l_{\text {atten }}$, as shown in Fig. 6, was varied to achieve four cases: total attenuation in the return path of: 1) $0 \mathrm{~dB}$; 2) $-8.75 \mathrm{~dB}$; 3) $-9.75 \mathrm{~dB}$; and 4) $-10.75 \mathrm{~dB}$.

An electron beam was defined with parameters as shown in Table II, and the time-varying fields were monitored at several locations within the simulated geometry. Discrete Fourier transforms (DFTs) were performed on the time-varying electric fields at a position 11 in the return path (see Fig. 6) to investigate how the excitation frequency evolved with time. Fig. 7(a) illustrates an overdriven case of large feedback with $0-\mathrm{dB}$ return path loss. The spectrum does not lock into any single frequency, and a broad multifrequency oscillation spectrum over the $528-580-\mathrm{GHz}$ regime is observed. When the return path loss is increased to approximately $-8.75 \mathrm{~dB}$, the oscillator appears to self-select two preferred frequency states around 520 and $580 \mathrm{GHz}$ [Fig. 7(b)]. When the loss is further increased to $-9.75 \mathrm{~dB}$, preferential oscillation in a single-frequency state at $580 \mathrm{GHz}$ is seen [Fig. 7(c)]. Finally, when the loss is increased to $10.75 \mathrm{~dB}$, there is no spontaneous signal growth, as the loss in the return path exceeds the forward gain.

\section{B. Time-Frequency Analysis}

When the (time-integrated) DFT spectrum shows multiple frequencies, it is of interest to know whether this represents unstable hopping between the frequencies or simultaneous generation of multiple frequencies. If stable multifrequency components are generated, one could use a filter system to suppress unwanted frequencies. If hopping between frequencies occur, it implies the need to back off on feedback to get stable oscillator output. DFTs do not provide the answer to


Fig. 7. Simulations showing frequency selection for the $560-\mathrm{GHz}$ oscillator. (a) 0-dB loss. Overdriven feedback having multifrequency oscillation states. (b) $-8.75-\mathrm{dB}$ loss. Intermediate feedback, showing selection of two preferred frequency states. (c) $-9.75 \mathrm{~dB}$ loss. Critical feedback showing approach to single-frequency state.

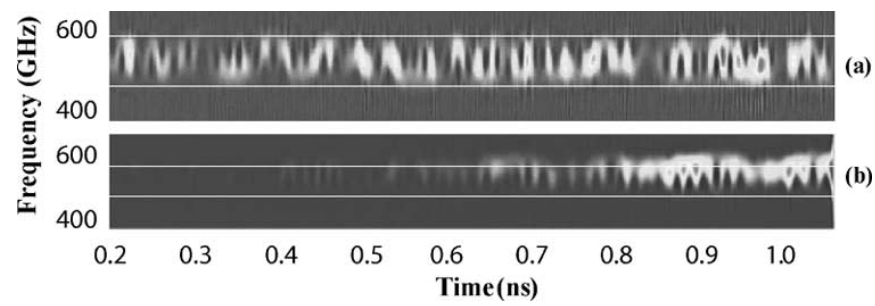

Fig. 8. Time-frequency analysis. (a) Intermediate feedback $(-8.75 \mathrm{~dB}$ return loss). (b) Critical feedback ( $-9.75 \mathrm{~dB}$ return loss).

this question of simultaneous multifrequency generation or hopping, particularly when the spectral evolution occurs on a relatively rapid timescale. As the time window is narrowed, one loses resolution and the information gets distorted. Joint time-frequency analysis eliminates the uncertainty of the windowed DFT [25]. Williams [26], [27] developed reduced interference distributions to provide high time-frequency resolution with minimization of cross terms and interference. Williams' binomial time-frequency distribution program was utilized earlier for the time-frequency analysis of modulation in high-power microwave sources [28]. In this study, it was used to analyze data from the MAFIA simulations for the -8.75 and $-9.75 \mathrm{~dB}$ cases of return path loss.

Fig. 8 shows the results for the intermediate feedback case of $-8.75 \mathrm{~dB}$ of return path loss [Fig. 8(a)] corresponding to Fig. 7(b). The instantaneous spectrum is observed to be rapidly hopping between two preferred frequency states at 520 and $580 \mathrm{GHz}$. For critical feedback at $-9.75 \mathrm{~dB}$ return path loss the frequency hopping is not observed. The oscillator is observed to have settled into one "preferred" state at $580 \mathrm{GHz}$ [Fig. 8(b)], corresponding to Fig. 7(c).

When Fig. 8(b) or Fig. 7(c) is described as single moded, it should be understood that this is a qualitative characterization. To achieve fully stabilized, single-moded behavior with a narrow line spectrum, it would have been necessary to run either many more simulations with very small changes in the return path attenuation or to run the simulations much longer, well into equilibrium. Neither of these options was feasible, due to the long run times required for these simulations. Therefore, it 


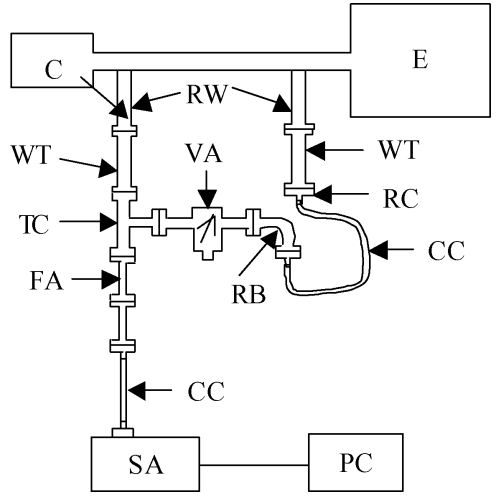

Fig. 9. Schematic of the experimental system for the 50-GHz FWG TWT oscillator. C: collector; E: electron gun; RW: rectangular waveguide (WR19); WT: waveguide transition (WR19 to WR22); RC: rectangular to coaxial adapter; CC: coaxial cable; RB: rectangular bend; VA: variable attenuator; TC: T coupler; FA: fixed waveguide attenuator; SA: spectrum analyzer; PC: computer.

was necessary, instead, to rely on the qualitative differences observed in the cases completed to illustrate the general nature of the dynamics. More precisely, it can be asserted that Fig. 7(c) is distinctly different than Fig. 7(b) in that there is a clear predominance of radiation in Fig. 7(c) at $580 \mathrm{GHz}$ compared to Fig. 7(b), which has two comparable intensity spectral peaks at approximately 520 and $580 \mathrm{GHz}$. Fig. 8(b), corresponding to Fig. 7(c), shows that, again, the spectral content strongly favors the 580-GHz state. Fig. 8(a), corresponding to Fig. 7(b), clearly is hopping between 520 and $580 \mathrm{GHz}$ with roughly equal time spent at each state. It is expected that very slightly increasing the return path loss would produce a simulation with a more stable single-frequency oscillation [than Fig. 7(c) or Fig. 8(b)]. However, the main point to draw from these simulations and analyses is that the two (or multiple) frequency oscillation behaviors associated with large feedback (low return path attenuation) involve frequency hopping, not stable, simultaneous oscillation at multiple frequencies, and that increasing the return path loss is indeed the primary "control" variable responsible for singleversus multiple-frequency operating states.

\section{EXPERIMENTAL SETUP AND METHODS}

The basic operating principles of a TWT oscillator with recirculated feedback are not known to have been previously reported. There are a number of important fundamental issues to investigate concerning oscillation thresholds and oscillation states. Since FWG TWTs can have up to $30 \%$ bandwidth, it is an interesting question to determine what frequency (or frequencies) will be self-selected in the saturated oscillation state. The fraction of output power that is fed back to the input is expected to affect whether the device operates below start oscillation, at critical oscillation equilibrium, or in an overdriven state.

To investigate the physics of the oscillator, a scaled experiment at $50 \mathrm{GHz}$ was carried out. Details on the conceptual design and fabrication of the 50-GHz FWG TWT amplifier can be found in [29]. The capability of FWG TWTs for forward-wave amplification with appreciable power and bandwidth has been previously demonstrated [29].

Fig. 9 shows a schematic of the experimental assembly. The amplifier has been converted into an oscillator by using an external feedback loop which consists of waveguide components, (a)

(b)


(c)
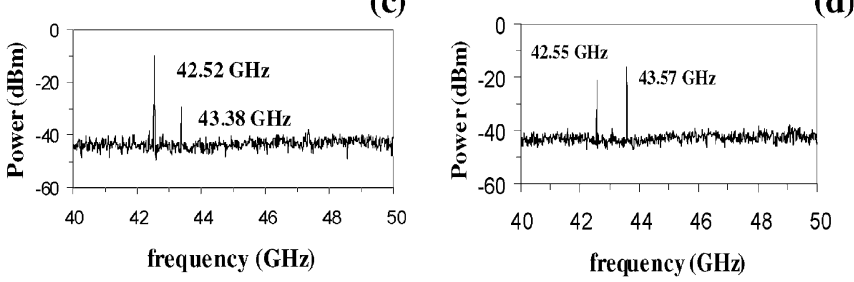

(d)

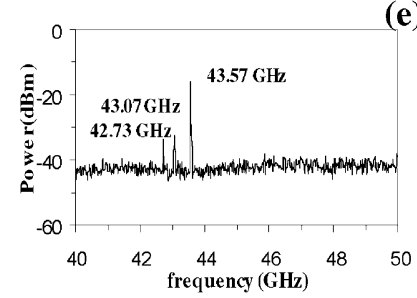

(e)

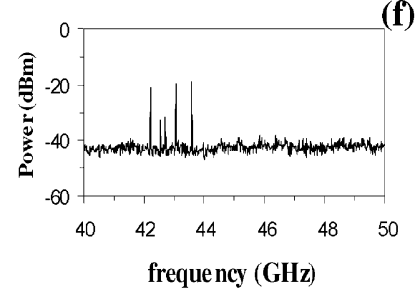

Fig. 10. Spectral evolution with feedback attenuation. Figures (a)-(f) represent different values of total feedback attenuation. (a) $-28.0 \mathrm{~dB}$. (b) $-26.0 \mathrm{~dB}$. (c) $-15.1 \mathrm{~dB}$. (d) $-8.25 \mathrm{~dB}$. (e) $-7.0 \mathrm{~dB}$. (f) $-2.5 \mathrm{~dB}$.

a variable attenuator to vary the level of output power fed back to the input, and a $\mathrm{T}$ coupler for coupling a fraction of the output power into a spectrum analyzer for measurement purposes.

The beam voltage and current are $20-21 \mathrm{kV}$ and $60-100 \mathrm{~mA}$, respectively. Measurements were made in a pulsed mode with $200-\mu$ s-long pulses with a repetition rate of 5 pulses/s. The generated frequency spectrum was observed using a HP $8565 \mathrm{E}$ $(30 \mathrm{~Hz}-50 \mathrm{GHz})$ gated spectrum analyzer.

\section{EXPERIMENTAL RESULTS}

\section{A. Spectral Evolution}

By varying the attenuation in the feedback leg using the variable attenuator, we observed the self-selected oscillation frequencies at different feedback power levels. The total attenuation in the feedback leg can be varied between $\sim-3$ and $-30 \mathrm{~dB}$ over the $40-50-\mathrm{GHz}$ range. This includes losses by all RF components and cables. At full attenuation, no oscillations are observed at any frequency [Fig. 10(a)]. The critical feedback threshold appears at $\sim-26 \mathrm{~dB}$ where stable single-frequency oscillations at $43.4 \mathrm{GHz}$ are observed [Fig. 10(b)]. The single-frequency oscillations persist until $-15.1 \mathrm{~dB}$ [Fig. 10(c)], where a second frequency at $42.5 \mathrm{GHz}$ appears. At $-8.25 \mathrm{~dB}$ feedback, the frequency at $43.4 \mathrm{GHz}$ has disappeared while a new oscillation at $43.6 \mathrm{GHz}$ appears along with the one at $42.5 \mathrm{GHz}$. At $-7.0 \mathrm{~dB}$, frequencies at 42.7, 43.1, and $43.6 \mathrm{GHz}$ appear [Fig. 10(e)]. Finally, at even lower levels of attenuation, no clear tendency is observed, and the spectrum consists of many frequency components in the range of $40-50 \mathrm{GHz}$ as indicated in Fig. 10(f). This observation 


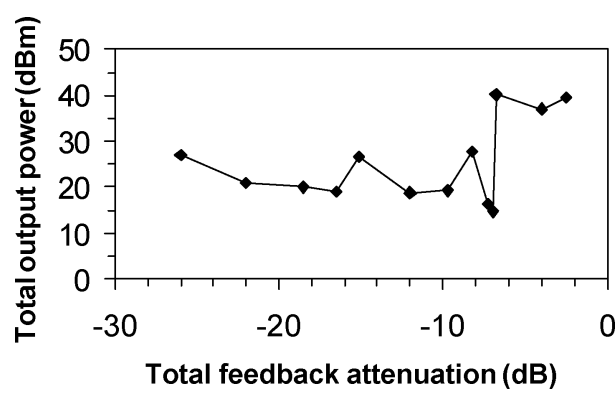

Fig. 11. Variation of the total spectral power with feedback attention.

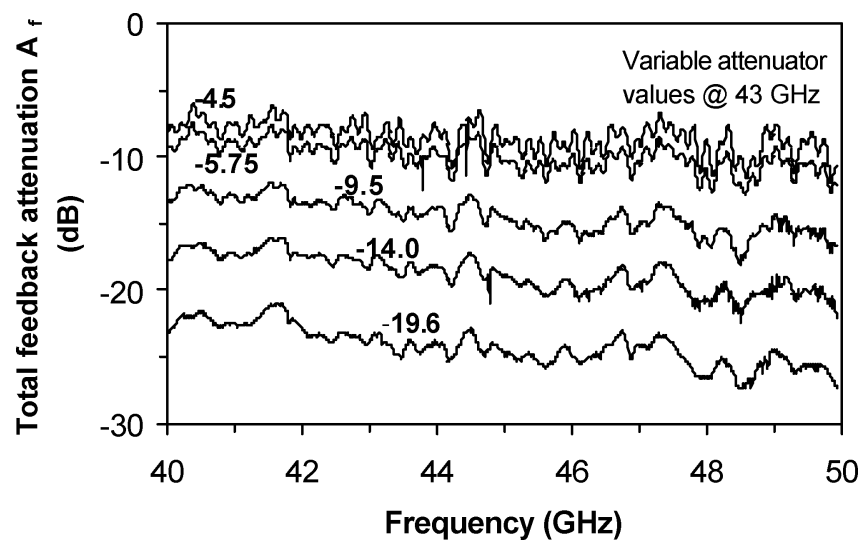

Fig. 12. Frequency response of the feedback circuit with the values of the variable attenuator as a parameter.

shows good qualitative agreement with the MAFIA simulations in Section II-A.

The total integrated spectral power $P_{T}$ (in $\mathrm{dBm}$ ) can be evaluated from

$$
P_{T}=\sum_{j} P\left(f_{j}\right)
$$

where $P\left(f_{j}\right)$ is the output power at a particular frequency $f_{j}$ that is distinctly visible above noise floor. Fig. 11 shows a plot of $P_{T}$ versus feedback attenuation. It is seen that the total generated power (measured at the exit of the TWT) varies between 0.03 and $10 \mathrm{~W}(15-40 \mathrm{dBm})$. The total power jumps at the attenuation changes where oscillations appear or disappear (see Fig. 10). For example, a sharp increase in power is seen at $-6 \mathrm{~dB}$ where many frequencies appear.

\section{B. Frequency Response of Feedback Leg}

The frequency response of the feedback circuit was measured using a Hewlett-Packard $83650 \mathrm{~L}$ swept continuous-wave $(\mathrm{CW})$ generator $(10 \mathrm{MHz}-50 \mathrm{GHz})$. The synthesizer was swept in the continuous mode in the range of 40-50 GHz. Fig. 12 shows the generated response of total feedback attenuation $A_{f}$ versus frequency for variable attenuation values of $-4.5,-5.75$, $-9.5,-14$, and $-19.6 \mathrm{~dB}$, respectively. With increase in frequency, $A_{f}$ shows a very slight, monotonic decrease, consistent with the theoretical dependence of loss with frequency for a constant resistivity

$$
\operatorname{loss} \propto e^{-\sqrt{\pi f \mu_{o} \sigma} z}
$$

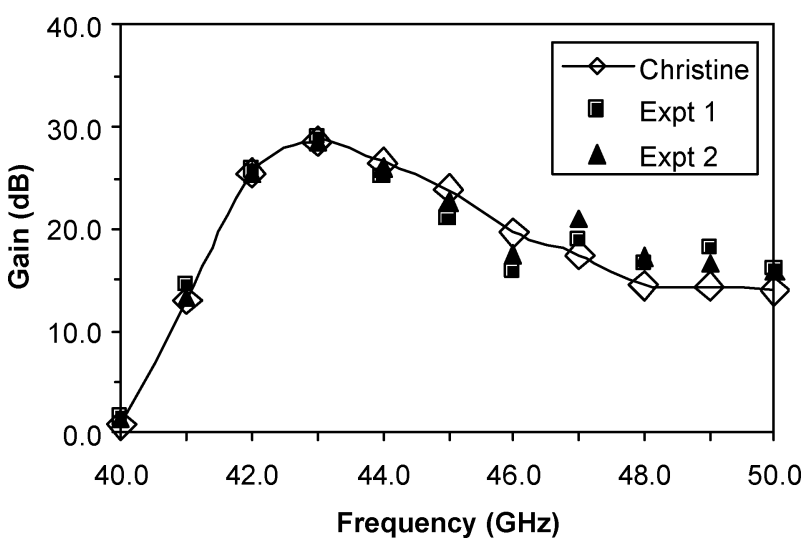

Fig. 13. Small signal gain of the 50-GHz FWG TWT amplifier. Results on two different runs of the experiment are shown. Notice the excellent agreement with CHRISTINE simulations at $20.75-\mathrm{kV}$ beam voltage and $60-\mathrm{mA}$ beam current.

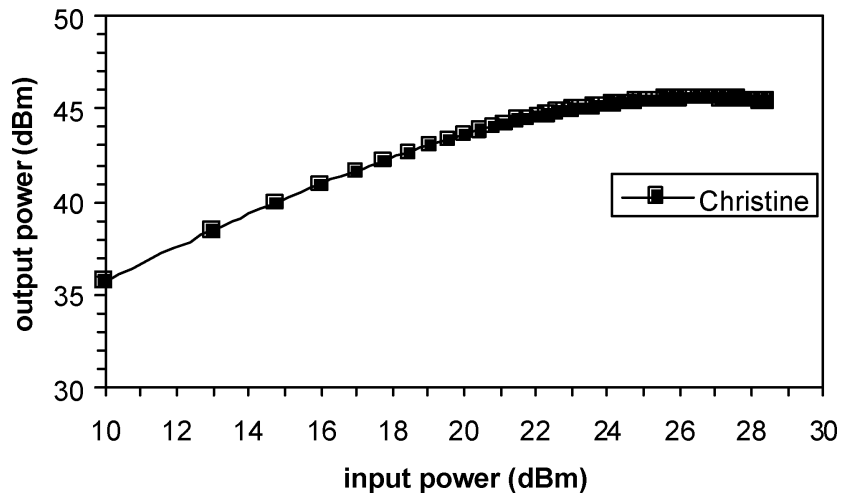

Fig. 14. Drive curve for the FWG TWT amplifier as simulated by CHRISTINE at $43 \mathrm{GHz}, 20.75-\mathrm{kV}$ beam voltage, and $60-\mathrm{mA}$ beam current.

where $f$ is the frequency, $\mu_{o}$ is the permittivity of free space, $\sigma$ is the wall conductivity (inverse of resistivity), and $z$ is the axial distance. This measurement established that the level of attenuation in the feedback circuit does not determine the oscillation frequency.

\section{Small Signal Gain and Cold Circuit Loss}

The small signal gain of the FWG TWT amplifier was measured in the frequency range of $40-50 \mathrm{GHz}$, in steps of $1 \mathrm{GHz}$. Fig. 13 shows the result on two different runs of the experiment, represented by dark triangles and squares. On the same plot shown by a line is the result of simulation for a beam voltage of $20.75 \mathrm{kV}$ and a beam current of $60 \mathrm{~mA}$. The simulations were conducted using CHRISTINE [24]. The maximum gain is about $27 \mathrm{~dB}$ at $\sim 43 \mathrm{GHz}$. Excellent agreement is observed between the experiment and simulation results. By shutting off the beam, the cold-circuit loss of the FWG TWT amplifier was measured in the above frequency range. This information was used in the simulations. The important conclusion was that the maximum small signal gain occurs between 42 and $44 \mathrm{GHz}$, precisely where the self-selected oscillations are observed.

D. Drive Curve: $P_{\text {in }}$ Versus $P_{\text {out }}$

Fig. 14 shows the CHRISTINE-simulated drive curve for the FWG TWT amplifier at $43 \mathrm{GHz}$ (location of maximum gain). Saturation is predicted to occur for input power levels 


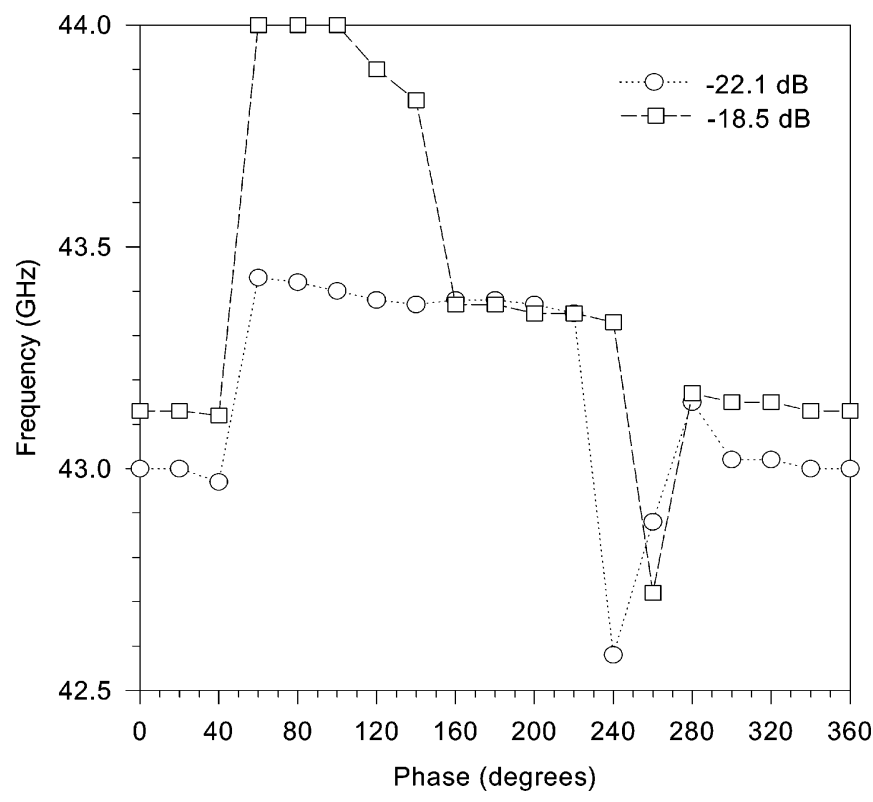

Fig. 15. Phase sensitivity of the generated oscillations.

of $\sim 25 \mathrm{dBm}$. The predicted saturated output power is $36 \mathrm{~W}$ $(\sim 46 \mathrm{dBm})$ and is greater than the experimentally measured maximum total output power of the oscillator $(10 \mathrm{~W})$ at $43 \mathrm{GHz}$. Experimental verification of the drive curve was not possible due to limitations on the maximum output power capability $(\sim 5 \mathrm{dBm})$ of the synthesizer. Predicting the maximum oscillator power (and efficiency) from the amplifier characteristics is a subject of continuing study. It is interesting to note that the small signal gain is $26 \mathrm{~dB}$, which is equal to the maximum attenuation that will allow a single-frequency oscillation. This is in agreement with theory and with MAFIA simulations for the $560-\mathrm{GHz}$ oscillator.

\section{E. Feedback Phase Stability of Oscillations}

To examine the stability of the oscillations $(42-44 \mathrm{GHz})$ to the feedback signal's phase, a $Q$ band $(33-50 \mathrm{GHz})$ phase shifter (Hughes $4752 \mathrm{H}-1000$ ) was placed in the feedback circuit. The phase of the feedback signal was varied from 0 to $360^{\circ}$ with respect to the input signal and changes in the oscillation spectrum were recorded.

Fig. 15 shows the sensitivity of the oscillations to changes in feedback phase. Measurements were made for cases where single-frequency oscillations were observed close to critical feedback $(-22$ and $-18.5 \mathrm{~dB})$. It is seen that the oscillations show some sensitivity to changes in phase $\varphi$ over $0-360^{\circ}$. At $-22.1 \mathrm{~dB}$ the frequency remains locked over ranges of $\varphi$ extending from 60 to $220^{\circ}$, while at other values of $\varphi$ there can be a change in the frequency of up to $1 \mathrm{GHz}$. This fact that the frequency does show sensitive dependence on the feedback signal's phase is a subject of continuing investigation.

\section{F. Some Implications of the Results of Modeling and Experiments}

Based on the preceding simulation and experimental observations, we may draw some inferences on the extension of FWG
TWTs to the THz regime. As an alternative to BWOs, there appear to be several intrinsic differences to be appreciated. First, as indicated by the two different beam lines in Fig. 2, to operate as a FWG BWO at the same frequency as the regenerative FWG TWT oscillator, it is either necessary to work at approximately five times higher voltage or significantly smaller pitch $p$ (for BWO operation in the first Brillouin zone) or else to operate at a higher space harmonic. In the latter case, one must accept a lower interaction impedance which will decrease the growth rate, increase the device length, and reduce intrinsic device efficiency.

However, even more interesting for the regenerative forward-wave oscillator configuration are potential opportunities for modulated signal generation. Other than "brute force" pin-diode amplitude modulation of the output, there are few options with a BWO besides modulation of the beam voltage or current. If thermionic cathodes are used, such beam modulations are necessarily low frequency, limited by parasitic capacitance in the electron gun. Moreover, they require either high-voltage control supplies or at least control supplies floating at high (cathode) voltage. In contrast, the results of our study of regenerative oscillators suggest several intriguing possibilities for signal modulations that deserve future investigation. For example, the results of Figs. 10 and 15 suggest bistable behaviors that could enable modulation schemes using an electronically controlled active element within the return path leg. For example, by operating close to the oscillation threshold on feedback attenuation, small modulations in the return path attenuation are expected to yield an amplitude modulated carrier, as one alternately exceeds or drops below the oscillation threshold attenuation level. Fig. 15 suggests another interesting possibility, in which small, rapid changes in the return path phase enable a bistable frequency modulation between two frequencies, $f_{1}$ and $f_{2}$ separated by $\sim 1 \mathrm{GHz}$. Digital encoding based on the frequency states is envisioned with $f_{1}=0$ and $f_{2}=1$. Although these modulation schemes might seem simplistic in comparison to techniques used below $100 \mathrm{GHz}$, one must keep in mind some of the costs and challenges expected of synchronous detection and demodulation schemes at $\mathrm{THz}$ frequencies. Indeed, one of the attractions of THz-based data transfer schemes is that with so much "bandwidth to spare," simplified and inexpensive modulation methods such as AM and bistable FM become much more practical than at millimeter-wave or microwave frequencies. The ultimate limit on symbol rates would be determined by the equilibration time of the oscillator, which is several round-trip transits, according to the simulations. This would correspond to $\sim 1 \mathrm{~ns}$ or faster for $\mathrm{THz}$ oscillator designs envisioned.

While the regenerative feedback oscillator configuration appears to offer more options for signal control than a BWO, the results thus far do not establish whether the maximum saturated efficiency available to single-pass amplificiation is available to the regenerative oscillator or whether stability constraints limit the oscillator efficiency to a lower value. Finally, the issues that are expected to be important for the $\mathrm{THz}$ device but are of lesser importance for a millimeter-wave device (e.g., the experiment at $50 \mathrm{GHz}$ ) include fabrication, beam transport, vacuum maintenance, and circuit losses. 


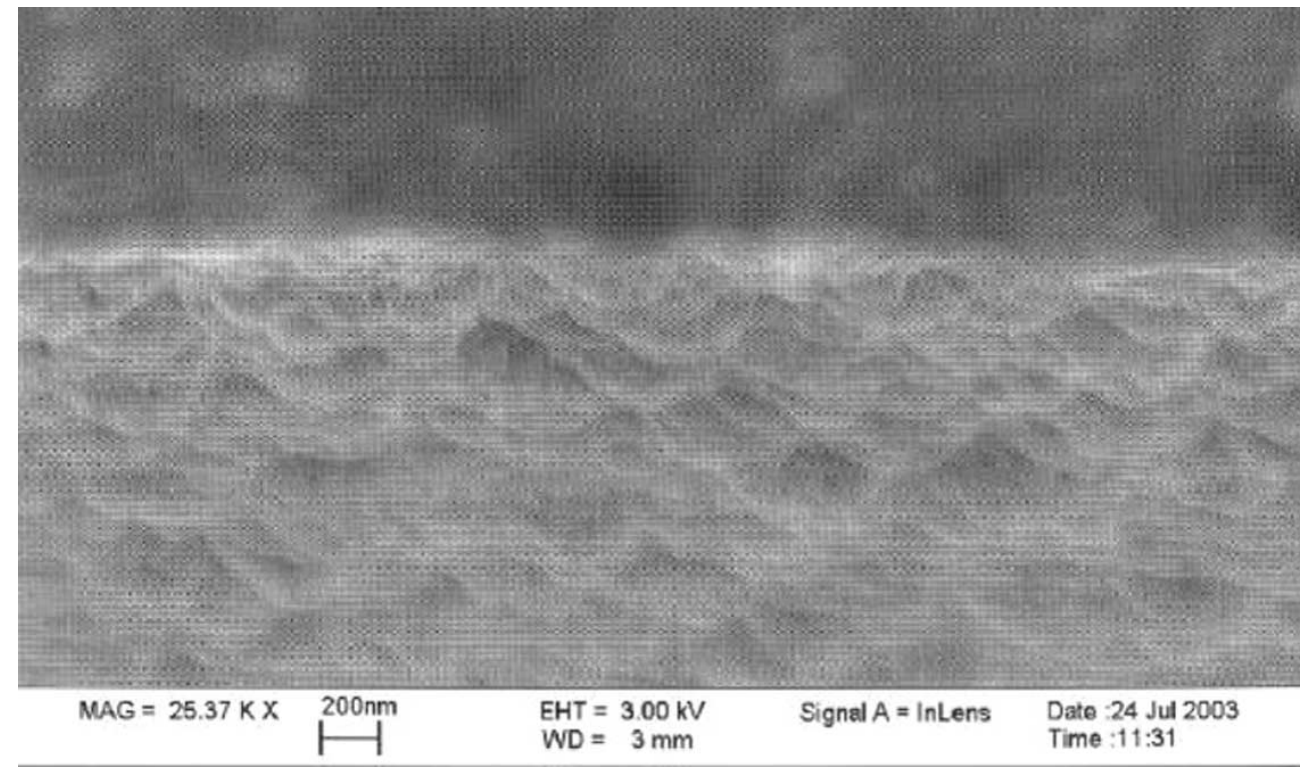

Fig. 16. SEM image of the edge of a LIGA-produced FWG TWT circuit. Note that the surface roughness is much less than $1 \mu \mathrm{m}$ (the indicator mark at the bottom of the image represents $0.2 \mu \mathrm{m})$.

\section{FABRICATION METHODS AND RESULTS}

\section{A. LIGA Microfabrication}

We have investigated two main methods of microfabrication namely lithography, electroforming, and molding (LIGA) and deep reactive ion etching (DRIE). LIGA is a German acronym standing for "Lithographic Galvonoformung $\mathrm{Ab}$ formung." In LIGA, three different techniques, depending upon the nature of irradiation, have been looked at, namely: 1) X-ray: polymethylmethacrylate (PMMA)/X-ray: PMMA; 2) UV:SU-8/X-ray:PMMA; and 3) UV:SU-8. In the first method, X-ray: PMMA/X-ray:PMMA, a low-aspect ratio (LAR) Au mask has been produced with X-ray irradiation of PMMA positive resist followed by resist development and $\mathrm{Au}$ electroplating [30]. The Au X-ray mask is used to expose a second high-aspect ratio (HAR) PMMA resist with X-rays to develop the HAR mold that will be filled by $\mathrm{Cu}$ electroplating. In the second technique, UV:SU-8/X-ray: PMMA, the LAR X-ray mask is produced by irradiating SU-8 negative photoresist with UV radiation followed by development and Au electroplating [31]. This X-ray mask is then used, as in the first case, for X-ray exposure of PMMA to produce the HAR PMMA mold for copper electroplating. The third process (UV : SU-8) involves a single step of exposing SU-8 with UV to produce a HAR mold for copper electroplate fabrication of the FWG circuit. Aspect ratio in this context is defined as the ratio between the serpentine wall height and the width. A typical example of a LAR mask feature would have an aspect ratio $1: 1$ or smaller. In contrast, a HAR mold feature's aspect ratio might be $4: 1$ or greater.

In the PMMA X-ray LIGA process [process 1)], graphite substrates were used. Experiments demonstrated that this provided more reliable adhesion to the PMMA than copper substrates. In exchange for better PMMA-to-substrate adhesion, using graphite substrates introduces an alternative complexity [32]. After filling the PMMA mold with electroplated $\mathrm{Cu}$, the

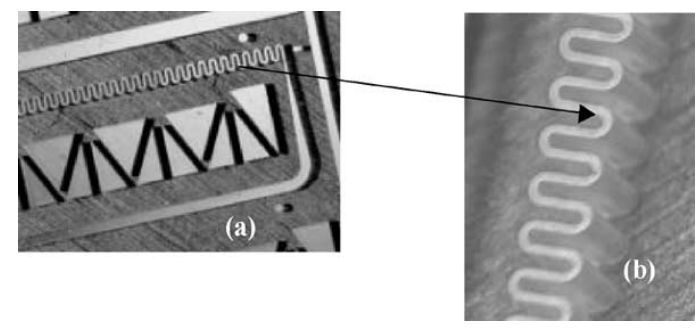

Fig. 17. (a) Illustrative PMMA electroplating mold obtained after X-ray exposure and resist development. (b) Magnified view of the FWG structure.

graphite substrate must be lapped away and replaced by additional electroplated $\mathrm{Cu}$. Fig. 16 shows a scanning electron microscope (SEM) image of the edge of a LIGA-produced FWG circuit. The important observation is that the surface roughness is much less than $1 \mu \mathrm{m}$ (the indicator mark at the bottom of the image represents $0.2 \mu \mathrm{m}$ ). This bodes well for realizing high conductivity copper walls desired for $\mathrm{THz}$ regime circuits with minimized losses. However, it still remains necessary to develop a procedure for fixturing and aligning two circuit halves to better than $10 \mu \mathrm{m}$, prior to bonding, as required for $400-\mathrm{GHz}$ and higher frequency circuits. Another challenge associated with this process is extended total process times, due to the combined requirements for thick PMMA resist layer coating, X-ray exposure times, and electroplating time.

In the LIGA process [process 2)], significant advantages were realized by significantly shortening the LAR X-ray mask development cycle time. In particular, the SU-8 photoresist has a much faster exposure time and obviates the need to wait for synchrotron time for X-rays. Fig. 17 shows an illustrative PMMA electroplating mold obtained after X-ray exposure and resist development. This specimen was obtained from an X-ray mask that had been developed using the UV : SU-8 exposure-and-development process. Some UV : SU-8 process optimization was required (e.g., optimizing the exposure dose) to minimize swelling in the exposed-and-developed 


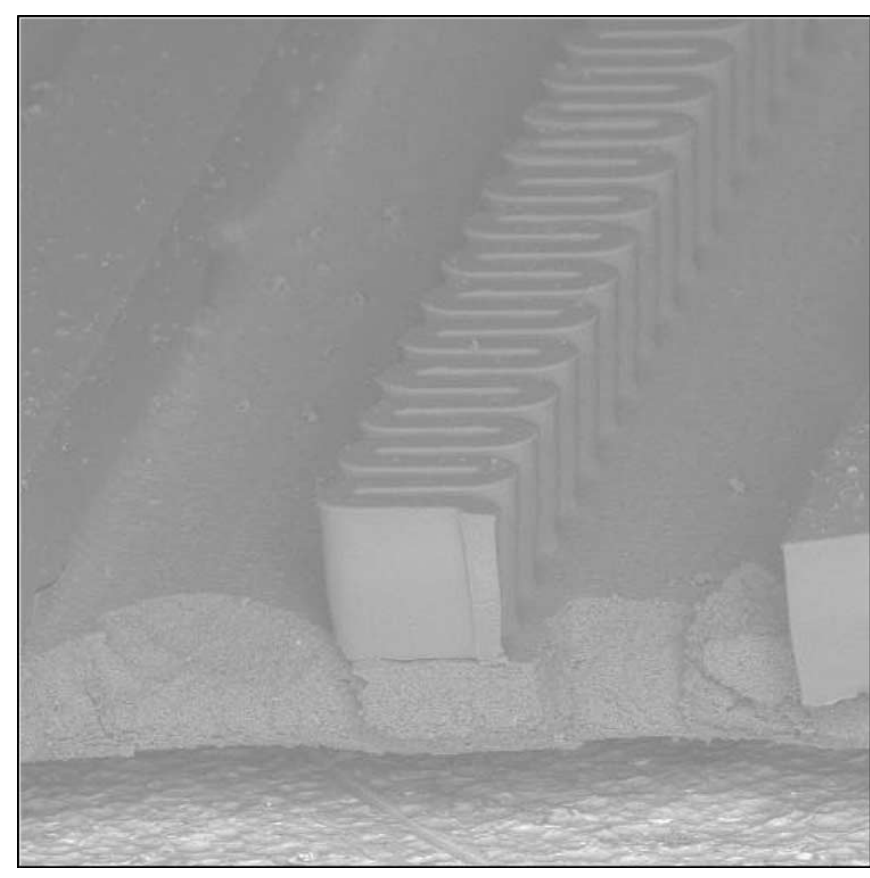

Fig. 18. HAR mold for copper electroplating with a $250-\mu \mathrm{m}$-high wall and a $100-\mu \mathrm{m}$ circuit pitch for a $400-\mathrm{GHz}$ FWG TWT circuit, produced completely by a UV : SU-8 process.

SU-8 structures. However, this optimization was relatively straightforward to achieve owing to the relatively quick process development cycle times.

Ultimately, developing the HAR copper electroplating mold solely with UV-exposed-SU-8 LIGA [LIGA process 3)] may offer an attractive, manufacturable process due to the relatively fast development cycle time and the lack of need for a synchrotron radiation source for X-rays. In an exploratory experiment, it was successfully demonstrated that a UV : SU-8 process could produce HAR molds for copper electroplating with dimensions and tolerances suitable for $400-\mathrm{GHz}$ FWG TWT circuits. Fig. 18 illustrates one such example with a $250-\mu \mathrm{m}$-high wall and a $100-\mu \mathrm{m}$ circuit pitch. The turn-to-turn uniformity was excellent (typical of photolithography-based fabrication). The next steps to produce a completed FWG circuit would be to flash evaporate a thin conductive sacrificial layer, such as aluminum, copper electroplate, and then separate mold and circuit by dissolving the sacrificial electrode layer.

\section{B. DRIE}

DRIE is an anisotropic reactive ion (plasma) process for microfabricating HAR structures in silicon through many repeated cycles of etching followed by passivating sidewalls via polymerization (creating a teflon-like film on the sidewalls). Typical etching masks are either $\mathrm{SiO}_{2}$ or PMMA, with common mask-to-silicon selectivity ratios of 100:1 (PMMA) and 150:1 $\left(\mathrm{SiO}_{2}\right)$, respectively. With the DRIE technique, one can produce serpentine waveguides either by etching serpentine trenches and coating them with conductor thin films or by using the etched Si structures as electroplating molds, similar to LIGA. As with LIGA, structures with a very high aspect ratio are possible and optimization can yield optically smooth sidewalls [33].
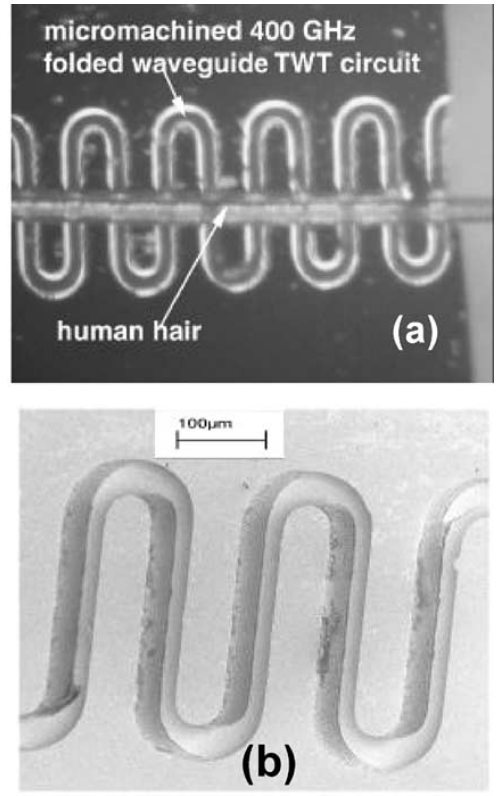

Fig. 19. Images of a prototypical $400-\mathrm{GHz}$ serpentine trench etched in $\mathrm{Si}$ using DRIE. These particular results were obtained using a 4- $\mu \mathrm{m}$-thick PMMA layer as both the photoresist and the etching mask (after resist development). (a) Optical microscope image. (b) SEM image.

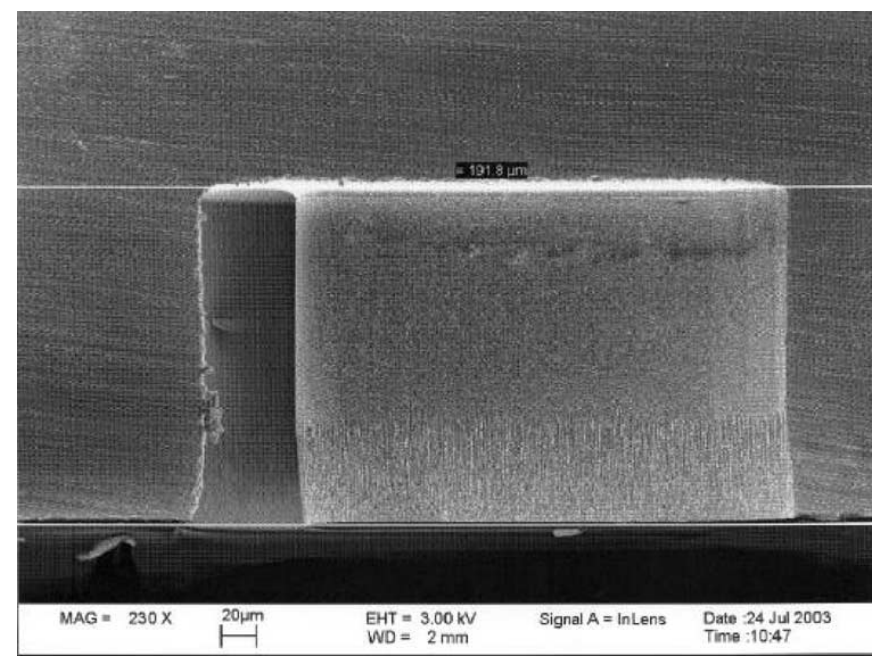

Fig. 20. SEM image showing the approximately $200-\mu$ m-deep trench obtained for the case shown in Fig. 19. The sidewall smoothness is better than $1 \mu \mathrm{m}$, similar to that of the LIGA example shown in Fig. 16.

Fig. 19 shows both optical microscope Fig. 19(a) and SEM images Fig. 19(b) of a prototypical 400-GHz serpentine trench etched in Si using DRIE. The process is relatively straightforward. These particular results were obtained using a $4-\mu \mathrm{m}$-thick PMMA layer as both the photoresist and the etching mask (after resist development). An approximately $200-\mu$ m-deep trench was obtained in this particular experiment, as shown in Fig. 20. Inside the trench, the sidewall smoothness was better than $1 \mu \mathrm{m}$, similar to that of the LIGA example shown in Fig. 16. Again, the tolerances for the circuit dimensions were excellent ( $\sim 1 \mu \mathrm{m}$ ), consistent with the use of a photolithographic process. Fig. 21 is a close-up view of a gold sputter-coated DRIE trench sidewall. In this view, the surface roughness inside the trench is revealed to be approximately $0.1 \mu \mathrm{m}$. Experiments 


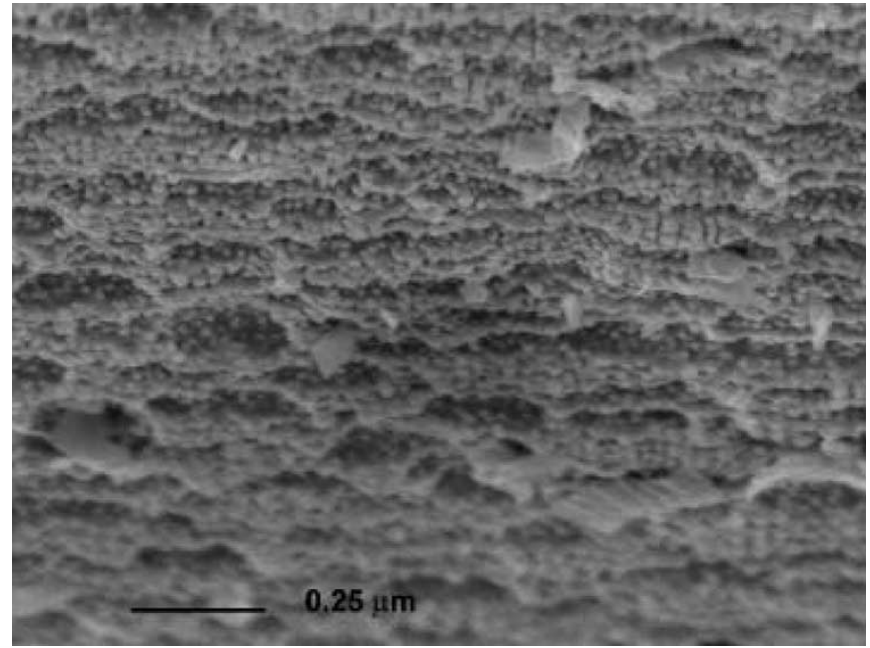

Fig. 21. Close-up view of a gold sputter-coated DRIE trench sidewall. Note that the surface roughness is revealed to be approximately $0.1 \mu \mathrm{m}$.

are underway to fabricate, align, and bond two such waveguide halves together and measure the electromagnetic waveguide properties. A significant advantage with the DRIE approach is that aligning two halves to within $\sim 1 \mu \mathrm{m}$ accuracy and bonding them should be feasible using commercially available $\mathrm{Si}$ wafer backside alignment and bonding techniques that are used extensively in $\mathrm{Si}$ microelectromechanical systems (MEMS) fabrication.

While all the methods of microfabrication appear to work, the desired method will be determined by many factors. Faster turn around and relatively quick development cycle times are a major requirement for large scale microfabrication. The all-X-ray LIGA process takes about one to three weeks to prepare masks and two to four weeks for completion of first electroplating. The necessity of a source for synchrotron radiation for X-rays can sometimes be a major hurdle. The all-UV : SU-8 technique appears to be the fastest, requires one to two days for preparation of masks and two to seven days for completion of first electroplating. Moreover, pattern transfer and resist adhesion were very good. This LIGA method does not require an X-ray source. The exposure can be done in-house with a UV source. On the other hand, the DRIE method allows one to directly produce serpentine waveguides either by etching serpentine trenches and coating them with conductor thin films or by using the etched $\mathrm{Si}$ structures as electroplating molds, similar to LIGA. Therefore, the all-UV : SU-8 and DRIE approaches are considered among the most promising technologies for fabricated THz TWT circuits.

\section{SUMMARY}

The THz regime of the electromagnetic spectrum provides immense research opportunities. However, it is a challenge to develop compact, powerful, efficient, and low-cost sources. Microfabricated FWG TWTs are a promising solution. The simulations and experiments have both demonstrated the capability of generating stable single-frequency oscillations using a delayed feedback amplifier configuration. Variation of the amount of recirculated power provides control over the stability of the oscillations. The results have identified oscillation thresholds, equilibria and nonstationary operating states, and the dominant factors influencing self-selected oscillation frequencies. The possibility of generating controlled single-frequency oscillations with appreciable power bears promise for generation of coherent radiation. It is important to know that the feedback leg does not determine oscillation frequencies; rather, frequencies with the highest gain are self-selected. This has an impact on amplifier design for future experiments. The feedback phase plays a limited but important role in the frequency selection effect.

The time-frequency analysis of a simulated $\mathrm{THz}$ oscillator provides further insights on frequency selection and oscillation dynamics. At intermediate feedback, the observed multifrequency spectra represent nonstationary, unstable hopping between different oscillation frequency states.

The regenerative forward-wave TWT oscillator may provide useful advantages over a BWO configuration with respect to possibilities for modulated carrier operation. Possibilities appear to include modulation of either the attenuation or phase in the return path leg. The former would provide bistable AM, while the latter would provide bistable FM. The modulation bandwidth limitation on either technique would probably be determined by the round-trip delay time, which is expected to be in the range of one to several gigahertz.

Two main microfabrication methods-namely, LIGA and DRIE- have been explored and remain under investigation. Among the several process options for LIGA, the all-UV : SU-8 seems to be most attractive, due to its fast development cycle times and its reliance on UV light sources rather than X-ray synchrotrons. The DRIE process is relatively straightforward and allows for direct fabrication of the serpentine trenches. With DRIE-produced circuit halves, one may use backside alignment and bonding popular with Si MEMS to realize $\sim 1 \mu \mathrm{m}$ accuracy in alignment.

\section{ACKNOWLEDGMENT}

The authors would like to thank D. Gallagher and colleagues at Northrop Grumman, Rolling Meadows, IL, for their helpful assistance.

\section{REFERENCES}

[1] P. H. Siegel, "Terahertz technology," IEEE Trans. Microwave Theory Tech., vol. 50, pp. 910-928, Mar. 2002.

[2] J. C. Wiltse, "Introduction and overview of millimeter waves," in Infrared and Millimeter Waves, K. J. Button, Ed. New York: Academic, 1981, vol. 4, ch. 1.

[3] "Terahertz sources and systems," presented at the NATO Advanced Research Workshop, R. E. Miles, P. Harrison, and D. Lippens, Eds., Chateau de Bonas, France, 2000.

[4] G. Chin, "Optically pumped submillimeter laser heterodyne receivers: Astrophysical observations and recent technical developments," Proc. IEEE, vol. 80, pp. 1788-1799, Nov. 1992.

[5] F. Capasso, C. Gmachl, D. L. Sivco, and A. Y. Cho, "Quantum cascade lasers," Phys. Today, vol. 55, pp. 34-40, May 2002.

[6] G. I. Haddad and R. J. Trew, "Microwave solid-state active devices," IEEE Trans. Microwave Theory Tech., vol. 50, pp. 760-779, Mar. 2002.

[7] N. R. Erickson, R. P. Smith, S. C. Martin, B. Nakamura, and I. Mehdi, "High efficiency MMIC frequency triplers for millimeter and submillimeter wavelengths," in IEEE MTT-S Int. Microwave Symp. Dig., vol. 2, 2000, pp. 1003-1006. 
[8] J. Harvey, E. R. Brown, D. B. Rutledge, and R. A. York, "Spatial power combining for high-power transmitters," IEEE Microwave, vol. 1, pp. 48-59, Dec. 2000.

[9] M. K. Hornstein, V. S. Bajaj, R. G. Griffin, K. E. Kreischer, I. Mastovsky, M. A. Shapiro, and R. J. Temkin, "Design of a $460 \mathrm{GHz}$ second harmonic gyrotron oscillator for use in dynamic nuclear polarization," in Proc. 27th Int. Conf. Infrared and Millimeter Waves Conf. Dig., 2002, pp. 193-194.

[10] S. J. Allen, "Terahertz, photon-assisted transport in semiconductor quantum structures," Nucl. Instrum. Methods Phys. Res. B: Beam Interact. Mater. At., vol. 144, pp. 130-134, Sept. 1998.

[11] P. H. Siegel, H. Manohara, J. Xu, B. Chang, A. Husain, and J. Hone, "Design and fabrication of a $1200 \mathrm{GHz}$ nanoklystron," presented at the 9th Int. Conf. THz Electronics, Charlottesville, VA, Oct 15-16, 2001.

[12] M. Chamberlain, J. Thorpe, J. R. Fletcher, R. E. Miles, E. Huq, and C. Mann, "Miniaturised vacuum technologies: Do they have a future for terahertz frequency devices?," in Proc. IEEE Pulsed Power Plasma Sci. (PPPS) Conf., 2001, pp. 160-168.

[13] G. Kantorowicz and P. Palluel, "Backward wave oscillators," in Infrared and Millimeter Waves, K. J. Button, Ed. New York: Academic, 1979, vol. 1, ch. 4.

[14] H. Bierman, "Microwave tube design efforts yield steady performance improvements," Microw. J., pp. 52-73, June 1988.

[15] J. East and G. Haddad, "Vacuum based sources for THz applications," presented at the 9th Int. Conf. THz Electronics, Charlottesville, VA, 2001.

[16] J. W. Hansen, “US TWT's from 1 to $100 \mathrm{GHz}$," Microw. J., pp. 179-193, 1989.

[17] C. Nilsen and M. Viant, "140 GHz traveling wave tube," in Proc. 4th Annu. Vacuum Electronics Review, 1995, pp. III-5-III-9.

[18] J. H. Booske, C. L. Kory, D. Gallagher, V. Heinen, K. Kreischer, D. W. Van der Weide, S. Limbach, P. Gustafson, W. -J. Lee, S. Gallagher, and K. Jain, "Terahertz-regime, micro-VEDs: Evaluation of micromachined TWT conceptual designs," in Conf. Rec. Abstracts IEEE Pulsed Power Plasma Science (PPPS) Conf., 2001, p. 161

[19] S. Bhattacharjee, C. L. Kory, W. -J. Lee, S. Gallagher, D. W. Van der Weide, J. H. Booske, and S. Limbach, "Comprehensive simulations of compact $\mathrm{THz}$ radiation sources using microfabricated, folded waveguide TWTs," in Proc. 3rd Int. Vacuum Electronics Conference (IVEC), 2002, pp. 26-27.

[20] S. Bhattacharjee, J. H. Booske, W. -J. Lee, C. L. Kory, S. Gallagher, M. Genack, D. W. Van der Weide, S. Limbach, M. R. Lopez, and R. M. Gilgenbach, "A compact folded waveguide traveling wave tube oscillator for the generation of terahertz radiation," Bull. Amer. Phys. Soc. (44th Annu. Meeting Division of Plasma Physics), vol. 47, p. 256, Nov. 2002.

[21] T. Weiland, "On the unique numerical solution of Maxwellian eigenvalue problems in three dimensions," Part. Accel., vol. 17, pp. 227-242, 1985.

[22] 3D EM simulation software for RF, wireless, packaging, and opto-electronic design. Ansoft HFSS. [Online]. Available: http://www.ansoft. com/products/hf/hfss/index.cfm

[23] D. M. MacGregor, Two Dimensional Nonlinear Multisignal Helix Traveling-Wave Tube Amplifier Computer Program. Ann Arbor, MI: Electrocon Int. Inc, 1993, vol. 1, User Manual.

[24] T. M. Antonsen Jr. and B. Levush, "Traveling-wave tube devices with nonlinear dielectric elements," IEEE Trans. Plasma Sci., vol. 26, pp. 774-786, June 1998.

[25] L. Cohen, Time Frequency Signal Analysis. New York: Prentice-Hall, 1995.

[26] W. J. Williams and J. Jeong, Time Frequency Signal Analysis: Methods and Applications, B. Boashash, Ed. New York: Longman \& Cheshire, 1992, ch. 3, pp. 74-98.

[27] W. J. Williams, "Reduced interference distributions: biological applications and interpretations," Proc. IEEE, vol. 84, pp. 1264-1280, Sept. 1996.

[28] C. W. Peters, R. L. Jaynes, Y. Y. Lau, R. M. Gilgenbach, W. J. Williams, J. M. Hochman, W. E. Cohen, J. I. Rintamaki, D. E. Vollers, J. Luginsland, and T. A. Spencer, "Time-frequency analysis of modulation of high power microwaves by electron-beam voltage fluctuations," Phys. Rev. E, Stat. Phys. Plasmas Fluids Relat. Interdiscip. Top., vol. 58, pp. 6880-6883, 1998

[29] G. Dohler, D. Gallagher, and J. Richards, "Millimeter wave folded waveguide TWTs," in Proc. Vacuum Electronics Annu. Review, 1993, pp. V15-V20.
[30] D. C. Mancini, N. Moldovan, R. Divan, F. DeCarlo, and J. Yaeger, "Process strategies for ultra-deep X-ray lithography at the advanced photon source," in Proc. SPIE, vol. 4557, 2001, pp. 77-84.

[31] R. Divan, D. C. Mancini, S. M. Gallagher, J. Booske, and D. Van der Weide, "Improvements in graphite-based X-ray mask fabrication for ultra-deep X-ray lithography," presented at the High Aspect Ratio Micro-Structure Technology Workshop (HARMST 2003), Monterey, CA.

[32] D. C. Mancini, N. Moldovan, O. V. Makarova, A. G. Peele, and T. H. K. Irving, "Use of graphite substrates for deep X-ray lithography," presented at the High Aspect Ratio Micro-Structure Technology Workshop (HARMST 2001), Baden-Baden, Germany.

[33] Surface Technology Systems [Online]. Available: http://www.stsystems.com

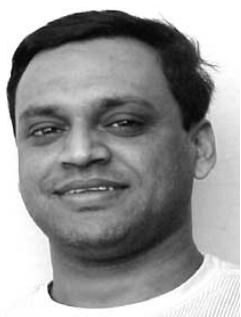

Sudeep Bhattacharjee (M'00) received the B.S. and M.S. degrees in physics from the University of North Bengal in 1989 and 1992, respectively, and the $\mathrm{Ph} . \mathrm{D}$. degree in experimental plasma physics from the Institute of Physical and Chemical Research (RIKEN), in affiliation with Saitama University, in 1999, on a Japanese Government Ministry of Education, Science and Culture (Monbusho) scholarship.

He was at the Institute for Plasma Research (IPR), first as a Graduate Research Scholar (1993-1994) and then as a Scientific Officer (1995-1996) under the Department of Atomic Energy, Government of India. From 1999 to 2001, he was a Distinguished Postdoctoral Fellow (Kisotokken) at the cyclotron center of RIKEN, where he did pioneering work involving development of a high-power microwave plasma ion source for nuclear physics applications, such as generation of heavy ions and multicharged radioactive ion beams. From 2001 to 2004, he was with the University of Wisconsin, Madison, as a Postdoctoral Research Associate. His research at the University of Wisconsin primarily involved development of electromagnetic sources of terahertz radiation and generation and control of chaos in traveling-wave tube amplifiers for communication and radar applications. As of October 2004, he joins the faculty at the Indian Institute of Technology-Kanpur as an Assistant Professor of Physics.

Dr. Bhattacharjee is a Member of the American Physical Society, the Physical Society of Japan, and the Japan Society of Applied Physics. He received the IEEE Early Career Travel Grant Award at the International Microwave Symposium (IMS03) in Philadelphia.

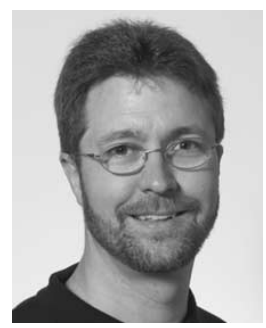

John H. Booske (S'82-M'85-SM'93) received the $\mathrm{Ph} . \mathrm{D}$. degree in nuclear engineering from the University of Michigan, Ann Arbor, in 1985.

From 1985 to 1989 , he was a Research Scientist at the University of Maryland, College Park. In 1990, he joined the faculty of the University of Wisconsin, Madison, where he is a Professor of Electrical and Computer Engineering. In 2001, he was appointed Director of the University of Wisconsin's Materials Science Program. His research interests include the experimental and theoretical study of coherent electromagnetic radiation sources and their applications, with emphasis in the RF, microwave, millimeter-wave, and terahertz regimes. Recent research activities include vacuum electronics, microwave and RF heating of materials, biological/biomedical applications of microwave and RF fields, high current density field emission cathodes, and ultrashallow junction fabrication in silicon CMOS transistors.

Prof. Booske received the University of Wisconsin Vilas Associate Award for research excellence and the U.S. National Science Foundation Presidential Young Investigator Award. He has been honored with many teaching awards, including the University of Wisconsin Chancellor's Distinguished Teaching Award and the Benjamin Smith Reynolds Award for Excellence in Teaching Engineering. He served as Codirector of a U.S. Department of Defense MURI99 consortium on innovative microwave vacuum electronics and as Director of a MURI04 consortium on cathode field emission and RF window breakdown in high-power microwave sources. 


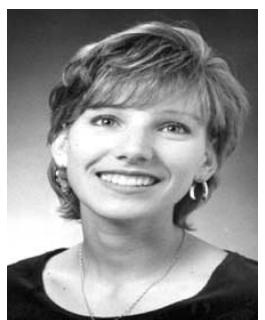

Carol L. Kory (M'95-S'97-M'02) received the B.S.E.E. degree from the University of Dayton, Dayton, OH, in 1992 and the M.S.E.E and Ph.D. degrees in engineering from Cleveland State University, Cleveland, OH, in 1997 and 2000, respectively.

From 1991 to 1992, she was with the NASA Glenn Research Center as an Intern Student with the Communication Technology Division, where her work involved analyzing traveling-wave tube slow-wave circuits using a three-dimensional particle-in-cell code. She became a full-time employee of the Analex Corporation, Cleveland, OH, in 1992, working with the same staff at NASA Glenn. Her work predominantly involves computational modeling, design, and development of vacuum electronics devices.

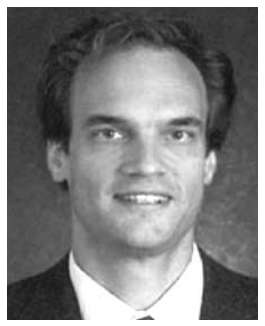

Dan W. van der Weide (S'86-M'86-S'89-M'92) received the $\mathrm{Ph} . \mathrm{D}$. degree in electrical engineering from Stanford University, Stanford, CA, in 1993.

He performed postdoctoral work at the Max Planck Institute. From 1995 to 1999, he was an Assistant and Associate Professor at the University of Delaware. He is currently Associate Professor of Electrical and Computer Engineering at the University of Wisconsin, Madison, where his group works in micromachined high-frequency electromagnetic sensors, localized microwave spectroscopy on biomolecules and semiconductor devices using multifunctional scanning probes and antennas, and terahertz spectroscopy with integrated antennas and nonlinear transmission lines.

Prof. van der Weide is a recipient of the National Science Foundation Presidential Early Career and Office of Naval Research Young Investigator Awards and is the Principal Investigator on a 2003 Air Force Office of Scientific Research Multidisciplinary University Research Initiative overseen by Lt. Col. G. Pomrenke, entitled "Nanoprobe Tools for Molecular Spectroscopy and Control."

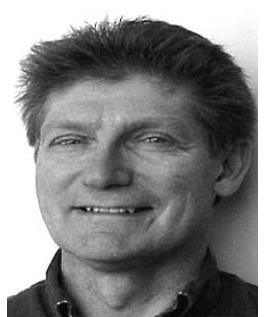

Steve Limbach received the B.S. degree from the University of Wisconsin, Madison (UW Madison), in 1973.

From 1974 to 1999 , he was a Researcher at the Laboratory of Molecular Biology at UW Madison. With a background in light and electron microscopy, he administered both the electron microscopy facilities and technical illustration facilities for the department. He also conducted research and was in charge of the light microscopy resources for Dr. G. G. Borisy. In 2000, he joined the laboratory of Prof. D. van der Weide in the Dapartment of Electrical and Computer Engineering, UW Madison, where he has conducted research and serves as the lab manager for the group. In the work presented here, he has assisted in instrumentation design and fabrication for the project.

S. Gallagher, photograph and biography not available at the time of publication.

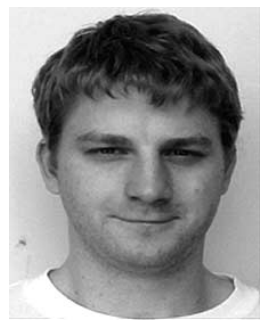

John D. Welter received the B.S.E.E. degree from the University of Wisconsin, Madison, in 2003. He is currently working toward the M.S.E.E degree at the University of Wisconsin, Madison.

In 2002, he was an Intern in the Electron Devices division, Northrop Grumman, where he worked on various projects involving traveling-wave tube amplifiers. His current research predominantly involves the use of microfabrication techniques for fabrication of miniature vacuum electronic devices.



Mike R. Lopez (M'04) received the B.S. degree in physics from Creighton University, Omaha, NE, and the M.S. and Ph.D. degrees in nuclear engineering from the University of Michigan, Ann Arbor, in 1998, 2002 , and 2003 , respectively.

$\mathrm{He}$ is presently focusing on z-pinch experiments and advanced diagnostics for the $\mathrm{Z}$ Accelerator and Z-Beamlet laser at Sandia National Laboratories, Albuquerque, NM.

Dr. Lopez is a member of the American Physical Society.

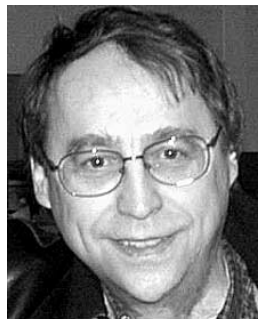

Ronald M. Gilgenbach (S'73-M'74-M'80-SM'92) received the B.S. and M.S. degrees from the University of Wisconsin, Madison, in 1972 and 1973, respectively (where he worked on electron cyclotron heating of mirror plasmas), and the Ph.D. degree in electrical engineering from Columbia University, New York, NY, in 1978.

He gained several years of industrial experience at Bell Laboratories, Holmdel, NJ. From 1978 to 1980, he was a Contractor at the Naval Research Laboratory (NRL), Washington, DC, where he was involved in research and development of the first long-pulse $35-\mathrm{GHz}$ gyrotron for plasma heating. He performed pioneering experiments on the ISX-B tokamak at Oak Ridge National Laboratory, Oak Ridge, TN, which represented the first gyrotron electron cyclotron heating experiments on a tokamak in the U.S. He joined the faculty of the University of Michigan, Ann Arbor, in 1980 and became Director of the Intense Energy Beam Interaction Laboratory, a post he still holds. He is currently a Professor with the Nuclear Engineering and Radiological Sciences Department, Applied Physics Program, University of Michigan. His research at Michigan has concentrated on the physics and applications of electron beams and microwaves, as well as laser-plasmas, laser diagnostics, and industrial materials processing. He has supervised 28 successful Ph.D. students and has authored over 90 published refereed articles. He has had research interactions with scientists at Air Force Research Laboratory Kirtland AFB, NM, Sandia National Laboratories, Albuquerque, NM, Northrop Grumman, Los Angeles, CA, General Motors Research Labs, Warren, MI, Los Alamos National Laboratory, Los Alamos, NM, Fermilab, Batavia, IL, and the NRL.

Dr. Gilgenbach previously served as Secretary and Member of the Executive Committee of the IEEE Plasma Sciences and Applications Technical Committee. He is a Fellow of the American Physical Society and received the 1997 Plasma Sciences and Applications Award from the IEEE PSAC.



R. Lawrence Ives ( S'83-M'83-SM'93) received the $\mathrm{Ph} . \mathrm{D}$. degree in plasma physics from North Carolina State University, Raleigh.

He began his microwave career in the Gyrotron Department at Varian Associates, Inc., Palo Alto, CA. In that position, he was responsible for designing electron guns, gyrotron circuits, collectors, and waveguide components. Gyrotrons are high power, high-frequency microwave and millimeter wave RF sources used for fusion research and industrial heating. He founded Calabazas Creek Research, Inc., which is involved in software development, microwave tube and component design, and uses of microwave power for environmental and heating applications. He was principle investigator in programs to develop a two-stage depressed collector system for Gaussian-mode gyrotrons at the 1-MW CW power level, $S$ band multiple beam electron gun for radar applications, a MW CW waterload for Gaussian mode gyrotrons, and a 15-kW CW $L$ band klystron for driving superconductor accelerator cavities. He is currently principle investigator on programs to implement field emission array cathodes into RF sources, multiple beam electron guns for high-power RF applications, improved magnetron injection guns for high power gyrotrons and gyroklystrons, a 50-MW multiple beam klystron, a microfabricated $W$ band traveling wave tube for satellite communications, and terahertz backward wave oscillators. He also provides consulting support to several commercial companies on electron gun and RF source development. 


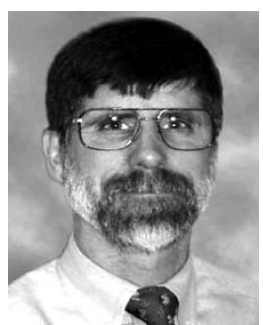

Michael E. Read (M'87) received the B.S., M.S. and $\mathrm{Ph} . \mathrm{D}$. degrees in electrical engineering and plasma physics in from Cornell University, Ithaca, NY, in 1970, 1972, and 1976, respectively. His graduate work was on the generation and propagation of intense relativistic electron beams (IREBs).

During 18 months of postdoctoral work at Cornell, he utilized IREBs for the generation of microwaves via space charge and cyclotron modes. He was a Member of the Department of Defense High Power Microwave Effects study group in 1982. Following his postdoctoral work, he worked at the Naval Research Laboratory (NRL) and from 1983 to 1986 was head of the Gyrotron Oscillators and Plasma Interactions Section of the Plasma Physics Division. While at NRL, he performed and managed fundamental research on electron cyclotron masers (gyrotrons), plasma heating, the effects of high-power microwaves on materials and systems, and the use of high-power microwave and millimeter-wave sources in applications, such as radar and EW. In 1986, he became a Principal Research Engineer with Physical Sciences, Inc., where he was responsible for research programs on microwave and electron beam generation and their applications. In 1992, he became Manager of the Electromagnetic Technology Area, overseeing work in microwave generation, atomic clocks, magnetic imaging and X-ray detection and imaging, and microwave driven plasma torches. He joined Calabazas Creek Research, Saratoga, CA, in 1999, where he is responsible for electron gun and high-power microwave device research. He has been a reviewer for the Department of Energy, the Air Force Office of Scientific Research, and the Office of Naval Research and has served as guest editor for the International Journal of Electronics. He is the author of over 50 papers in refereed journals and a contributor to two books. He also is the author of three patents, including one on a cavity commonly used in gyrotrons and another on a plasma torch. Recent work includes novel electron guns for a sheet beam klystron and gyroklystrons.

Dr. Read is a member of the American Physical Society.



Ralu Divan received the M.Sc. and Ph.D. degrees in chemistry from the Department of Chemistry, University of Bucharest, in 1977 and 1999, respectively.

From 1977 to 1995, she was with ICCE (Research Institute for Electronic Components)—Bucharest. From 1995 to 1999 , she was with the Romanian National Institute of Microtechnology (IMT). Since 1999, she has been with the Argonne National Laboratory, Argonne, IL. Her major areas of expertise are MOS technological processes, development of advanced optical resist processes, and microfabrication technologies. Her actual duties in Argonne National Laboratory are related to the development of soft X-ray lithography for high aspect ratio zone plates and ultradeep X-ray lithography for lithography-electroplating-molding (LIGA) applications. She has published over 60 papers in journals and conference proceedings relating to optical lithography, microelectromehcanical systems, and X-ray lithography.

D. C. Mancini, photograph and biography not available at the time of publication. 